\title{
1,1'-Carbonyldiimidazole and Mechanochemistry: A Shining Green Combination
}

\author{
Thomas-Xavier Métro,*® Jean Martinez, and Frédéric Lamaty* \\ Institut des Biomolécules Max Mousseron (IBMM), UMR 5247, CNRS, Université de Montpellier, ENSCM, Campus Triolet, Place \\ Eugène Bataillon, 34095 Montpellier cedex 5, France
}

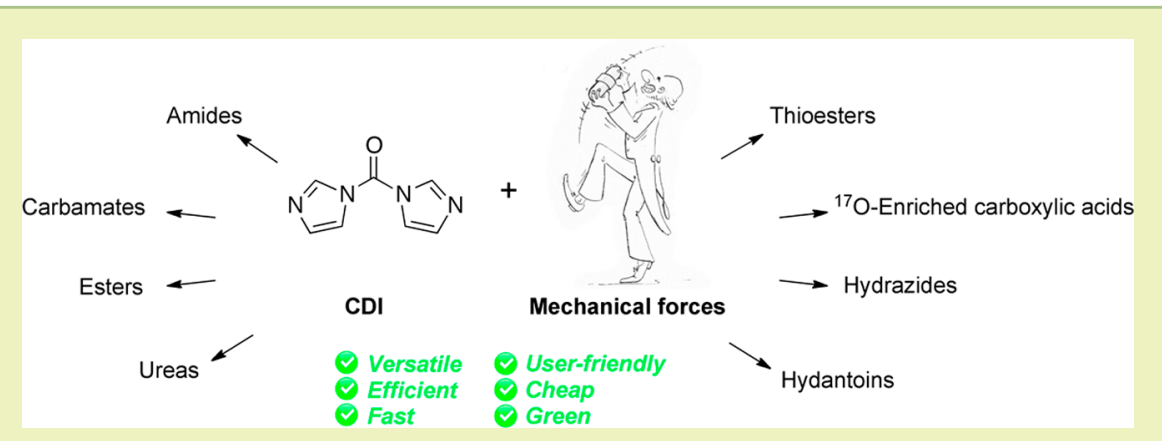

ABSTRACT: The application of mechanical forces to enable a chemical reaction, also known as mechanochemistry, is actually experiencing an impressive renewal of interest among the chemists community. One major advantage of this approach in organic synthesis lies in the possibility to run essentially solvent-free reactions. In recent years, some research groups have combined these types of solvent-free processes with the use of an eco-friendly reagent: 1,1'-carbonyldiimidazole (CDI). Thus, a wide range of molecules, including carboxylic acids, amines, and alcohols, could react efficiently with CDI under ball-milling conditions. By providing a vast array of products (amides, carboxylic acids, esters, ureas, hydantoins, etc.) in an efficient, fast, user-friendly, and green manner, this approach presents all the prerequisites to soon become a first choice methodology for all organic chemists. The reasons for the past and future successes of this combination are summarized, analyzed, and discussed herein.

KEYWORDS: CDI, 1,1'-Carbonyldiimidazole, Mechanochemistry, Ball-milling, Green chemistry, Sustainable chemistry, Solvent-free

\section{INTRODUCTION}

Although enabling the synthesis of an incredibly wide scope of molecules presenting exceptional complexity, organic synthesis generally suffers from the large amounts of toxic waste that are produced during syntheses. ${ }^{1}$ Defining green metrics to evaluate the environmental impact of chemical processes has clearly revealed solvents as major contributors to the production of undesirable waste. ${ }^{2}$ Yet, performing organic synthesis with less or no solvent in conventional batch reactors generally lead to material diffusion limitations. In order to solve these problems, some organic chemists have turned to the use of ball-mills. Thus, elevated mechanical forces could be applied to reaction mixtures thereby allowing for efficient mixing and reactivity of the reagents, isolation of products in high yields, reduction of the environmental impact and discovering unexpected selectivities. $^{3,4}$ Among the wide number of reactions that could benefit from this innovative approach, a reagent, 1,1'carbonyldiimidazole (CDI), stood out by showing particular efficiency while generating noticeable interest from various research groups.

\section{DISCUSSION}

Since its very first synthesis in $1957,{ }^{5,6} 1,1^{\prime}$-carbonyldiimidazole (CDI) has been used for various applications in organic synthesis. Due to its electrophilic nature, it has mainly been reacted with carboxylic acids, alcohols, and amines. ${ }^{7}$ The safety of its side-products, namely, imidazole and carbone dioxide, are of particular interest in an era in which environmental impact is of increasing significance. Formation of 1 or 2 equiv of imidazole as a side-product also opens the possibility to avoid the use of an external base whenever required. To the best of our knowledge, the first use of CDI under solvent-free conditions was reported in 2012 and was applied to the production of acyl-imidazoles, carboxylic imidazole esters, amides, and carbamates. ${ }^{8}$ Yet, the use of a spatula to mix the reagents prevented scale-up perspectives, and no control experiment was reported to exclude the possibility that formation of the intermediate and products could occur during the recovery with EtOAc. ${ }^{9}$ The same year our group showed that carboxylic acids could be easily activated by CDI by using a planetary ball-mill (pbm), ${ }^{10}$ thereby demonstrating this approach could be easily scaled up. ${ }^{11}$ Of note, the proof that starting materials reacted during the milling treatment was obtained by sampling aliquots that were not solubilized before being analyzed by IR. After activation of carboxylic acids with CDI, the acyl-imidazole intermediates were reacted with various $\mathrm{N}$-, $\mathrm{O}$-, $\mathrm{S}$-, and $\mathrm{C}$-nucleophiles, furnishing the pure correspond- 
ing products in high yields. ${ }^{12}$ These latters could be recovered by filtration using only water, thereby avoiding the use of any organic solvent from reaction to product recovery. No further purification (by crystallization or column chromatography) was necessary. Besides, the acylation reactions were proved to be faster than when performed in solution, while presenting a sharply reduced environmental impact, both in terms of volume and toxicity of produced waste. ${ }^{12}$ In addition, this protocol circumvented the use of dry organic solvent and nitrogen atmosphere frequently employed during synthesis in solution. As a representative and successful example of this approach, Teriflunomide, an API indicated in the treatment of multiple sclerosis, ${ }^{13}$ was isolated in $81 \%$ overall yield (Scheme 1 ).

Scheme 1. CDI-Mediated Synthesis of Teriflunomide ${ }^{a}$

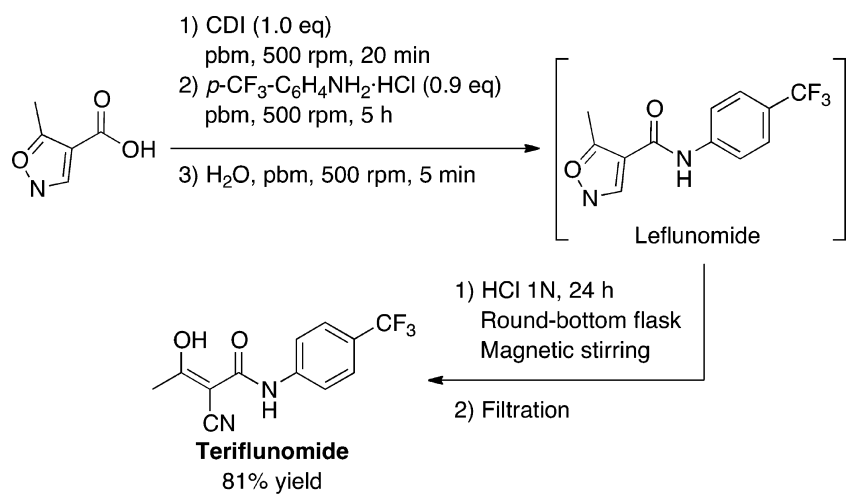

${ }^{a}$ Reproduced from ref 10 with permission from the Royal Society of Chemistry.

This approach was further applied by various research groups to the synthesis of a large array of benzoylhydrazides, ${ }^{14}$ hydroxamic acids, ${ }^{15}$ and glycoamides, ${ }^{16}$ with strong emphasis on the efficiency, user-friendliness, and low environmental impact of the method. Of note, the capacity of this strategy to avoid traditional solubility and purification concerns was highlighted. ${ }^{15,16}$ More recently, this approach was applied to the production of ${ }^{17} \mathrm{O}$-enriched carboxylic acids in view of analysis by ${ }^{17} \mathrm{O}$ NMR spectroscopy. ${ }^{17}$ Carboxylic acids were first activated by reaction with CDI in a vibrating ball-mill (vbm) to furnish the corresponding acyl-imidazoles. These intermediates were further hydrolyzed by ball-milling in the presence of small amounts of ${ }^{17} \mathrm{O}$-enriched water (Scheme 2).

Scheme 2. Carboxylic Acid ${ }^{17}$ O-Labeling by Activation with CDI

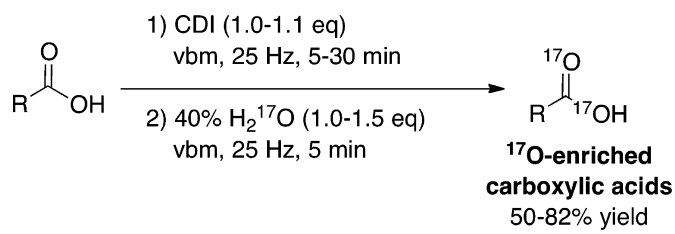

The corresponding isotopically enriched carboxylic acids were isolated in 50-82\% yields after classical workup. Compared to more conventional solvent-based labeling schemes, this protocol enabled the production of ${ }^{17} \mathrm{O}$-enriched carboxylic acids in a very efficient, user-friendly, and low-cost manner. By drastically lowering the required amounts of the costly ${ }^{17} \mathrm{O}$-enriched water, this mechanochemical approach opens an avenue for further research based on ${ }^{17} \mathrm{O}$ NMR spectroscopy.

Besides, CDI leads to the production of carbamates when reacted with an alcohol and an amine. Thus, ball-milling alcohols with CDI furnished carboxylic imidazole esters that reacted efficiently with amines to furnish the corresponding carbamates in high yields. This strategy could be applied to the synthesis of various $\mathrm{N}$-Z-, $\mathrm{N}$-Alloc- and $\mathrm{N}$-Moc-protected amino esters, while avoiding the use of problematic and more frequently used isocyanates and/or chloroformates. ${ }^{18}$ Besides, ball-milling amines with CDI furnished carbamoyl imidazole intermediates that were transformed to unsymmetrical ureas after successive addition of a second amine. These unsymmetrical ureas cyclized in situ to furnish hydantoins in high yields (Scheme 3). ${ }^{19-21}$

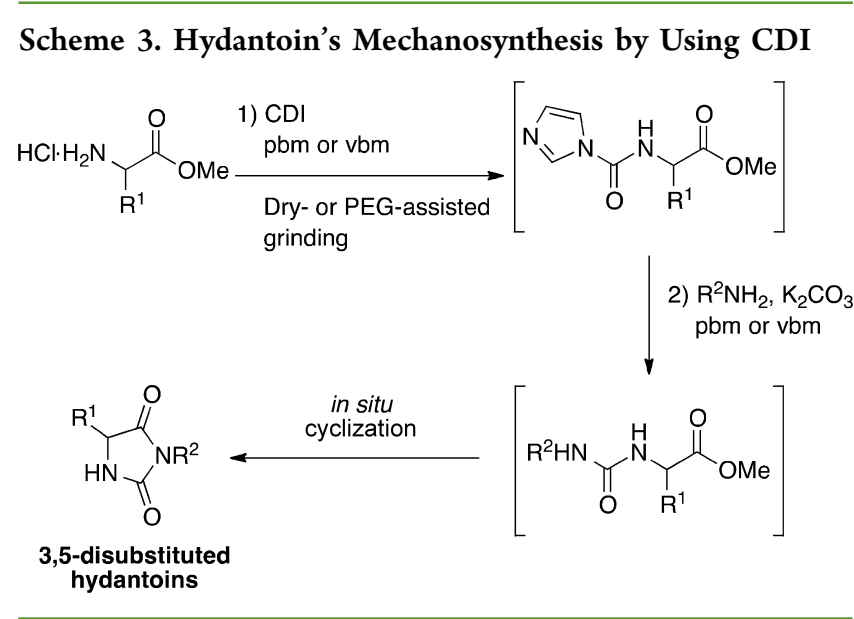

An alternative route to these hydantoins could be achieved by reacting dipeptides of general formula TFA.H-AA $-\mathrm{AA}_{2}-\mathrm{OR}$ with $\mathrm{CDI}^{20}$ Of note, PEG derivatives were identified as safe and eco-friendly liquid grinding assistants for the production of these hydantoins. ${ }^{21}$ When compared to conventional syntheses of hydantoins, combining the use of CDI and ball-milling generally furnished hydantoins in a more efficient and faster manner, while avoiding conventional harsh conditions.

Overall, many reasons can explain the success of combining the use of CDI and mechanochemistry. First, CDI is a white crystalline solid available in kilogram quantities, and its stability under atmospheric conditions is sufficient to avoid tedious and time-consuming precautions. ${ }^{22}$ It is cheap, and both CDI and its byproducts imidazole and carbon dioxide are safe. While $\mathrm{CO}_{2}$ is efficiently evacuated from the reaction mixture due to its gaseous nature, it is worth noting that imidazole can easily be removed by simple aqueous washings. These properties were advantageously utilized, in combination with ball-milling, for the production and isolation of products otherwise difficult to isolate. $^{15,16}$ These properties also enabled to produce fine organic molecules without any organic solvent from reaction to product recovery. ${ }^{10,12}$ Besides, it can be hypothesized that the relatively low melting point of imidazole $\left(\mathrm{mp} 88-91^{\circ} \mathrm{C}\right)$ favors the formation of eutectics. This may avoid reagents diffusion limitations that sometimes happen during solvent-free ballmilling. ${ }^{23}$ The formation of such eutectics is most probably the main reason why the majority of the studies mentioned herein do not report the use of external liquid grinding assistants. Similarly, production of gaseous $\mathrm{CO}_{2}$ may certainly help in mixing the reagents with efficiency. In cases where production 
of $\mathrm{CO}_{2}$ is a driving force for the reaction, the absence of an organic solvent may also favor the release of $\mathrm{CO}_{2}$ and therefore accelerate the reaction speed. ${ }^{24}$ While the lower reactivity of CDI compared to similar reagents (coupling agents, isocyanates, chloroformates) has often been mentioned as a disadvantage in solution, ${ }^{25}$ its utilization under solvent-free conditions is certainly filling this gap.

\section{CONCLUSION}

The versatile reactivity of CDI leads to the production of an exceptionally wide array of organic chemicals of high added value. Besides, the intrinsic properties of CDI makes it very adapted for applications in ball-milling. For these reasons, one can easily foresee that associating CDI and ball-milling will undoubtedly gain further popularity among the chemists community aiming at improving both efficiency and environmental impact of their chemical enterprise. As for CDI, the development of reagents taking into account their solvent-free, sustainable, and practical use in ball-milling will certainly contribute to the development and dissemination of green organic mechanosynthesis.

\section{AUTHOR INFORMATION}

\section{Corresponding Authors}

*E-mail: thomas-xavier.metro@umontpellier.fr.

*E-mail: frederic.lamaty@umontpellier.fr.

\section{ORCID}

Thomas-Xavier Métro: 0000-0003-2280-3595

\section{Notes}

The authors declare no competing financial interest.

\section{Biographies}

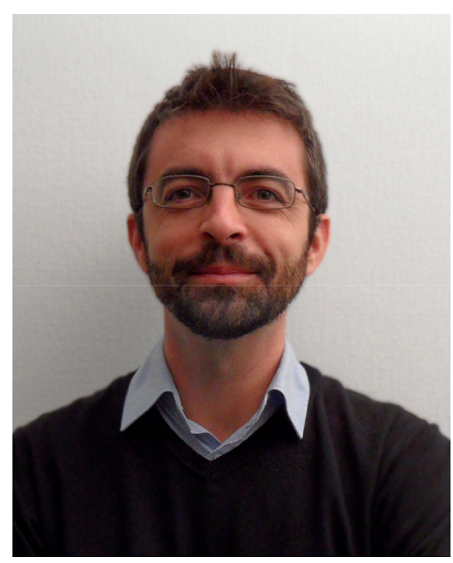

After having spent one year at Atofina Chemicals, USA, working on organic peroxides safety process research, Thomas-Xavier Métro graduated with a Master's degree in general chemistry from the Ecole Supérieure de Chimie Organique et Minérale and a Master's degree in medicinal chemistry from the Universite de Pharmacie de Montpellier both in 2004. He achieved his Ph.D. in organic chemistry in 2008 from the Université Pierre et Marie Curie (Paris) under the supervision of Dr. Domingo Gomez Pardo and Prof. Janine Cossy, working on the rearrangement of amino alcohols and its application to the synthesis of $(S, S)$-reboxetine and SSR241586. After a first postdoc in process R\&D at Sanofi Chimie in Sisteron and a second postdoc in medicinal chemistry at Sanofi-Aventis R\&D in Montpellier, he joined the CNRS in 2011 as a Chargé de Recherche in the Green Chemistry and Enabling Technologies Team at the Max Mousseron Institute for Biomolecules (IBMM; Montpellier). Since then, his research activity is mainly based on the development of solvent-free/solvent-less methodologies, aiming to provide the organic chemist with a set of solutions to reduce the environmental impact of his chemical enterprise.

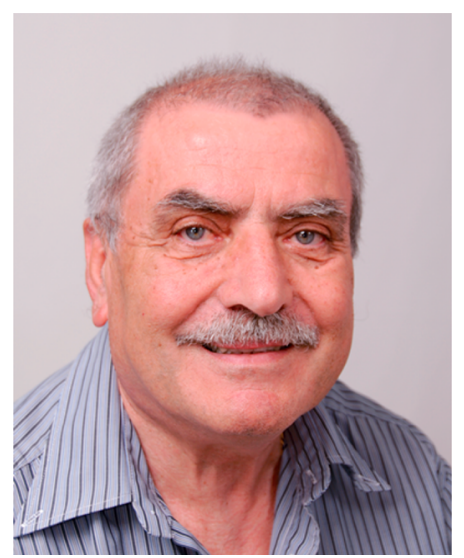

Jean Martinez received his Ph.D. degree in organic chemistry from the University of Montpellier at the Ecole Nationale Supérieure de Chimie de Montpellier under the direction of Prof. F. Winternitz. He completed his chemical education as a postdoctoral fellow with Dr. E. Bricas, Orsay, University of Paris Sud, and then with Prof. M. Bodanszky, Case Western Reserve University, Cleveland, OH. Back in France, he was recruited as a CNRS research associate and then as a research director in Montpellier. Pursuing his investigation at the interface of chemistry and biology, he joined the University of Montpellier and was appointed a Full Professor in both organic chemistry and medicinal chemistry. He became successively the head of various research laboratories in Montpellier including the Laboratory of Chemistry and Pharmacology of Biologically Interesting Molecules, the Laboratory of Amino Acids, Peptides and Proteins. He founded the Institute of Biomolecules Max Mousseron in 2007 and acted as the director until December 2014. In 2015, in the same institute he stayed as the head of the Department of Amino Acids, Peptides and Proteins. His current research interests focus on peptide chemistry, stereoselective syntheses of biomolecules, design and production of original biomaterials, green chemistry, and biology and pharmacology of neuropeptides of the gastrointestinal tract.

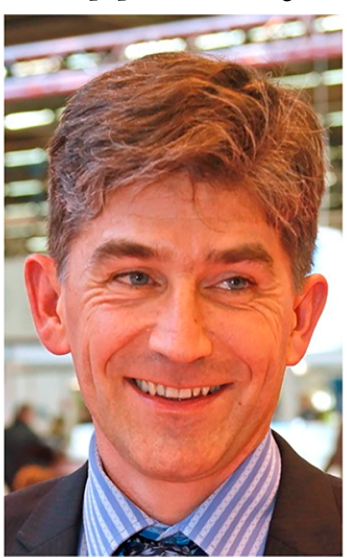

Frédéric Lamaty graduated as a chemical engineer in 1988 from the Ecole Supérieure de Chimie Industrielle de Lyon (ESCIL now ESCPE, France). In 1992, he received his Ph.D. from Purdue University (West Lafayette, IN, USA) under the supervision of Professor Ei-chi Negishi in the field of Pd-catalyzed cyclizations. He then joined, as a RhônePoulenc postdoctoral fellow, the group of Professor Marc Julia at the Ecole Normale Supérieure in Paris to work on the synthesis of vitamin A. In 1994, he obtained a permanent position at the Centre National de la Recherche Scientifique (CNRS) in Montpellier and is currently 
working as Directeur de Recherche at the Max Mousseron Institute for Biomolecules (IBMM). Since 2011, he is heading the Green Chemistry and Enabling Technologies team (www.greenchem.um2. fr) at IBMM. His research topics in the area of catalysis, organic, and green chemistry are devoted to the synthesis of amino acids, peptides, and heterocycles, the use of alternative solvents (PEG, PEG-IL, glycerol, water), and enabling technologies (microwaves, ultrasounds, ball-milling, continuous flow) with an emphasis on mechanochemistry. He was awarded in 2011 the Prix des Techniques Innovantes pour l'Environnement ADEME-Pollutec for his activity in peptide mechanosynthesis in solvent-free conditions.

\section{ACKNOWLEDGMENTS}

Université de Montpellier, Centre National de la Recherche Scientifique (CNRS), and LabEx CheMISyst (through ANR programme ANR-10-LABX-05-01) are acknowledged for financial support. The abstract graphic is partially reproduced from ref 23 with permission from the Royal Society of Chemistry. ${ }^{23}$

\section{ABBREVIATIONS}

CDI, 1,1'-carbonyldiimidazole; pbm, planetary ball-mill; vbm, vibrating ball-mill

\section{REFERENCES}

(1) Sheldon, R. A. The E factor 25 years on: the rise of green chemistry and sustainability. Green Chem. 2017, 19, 18-43.

(2) Constable, D. J. C.; Curzons, A. D.; Cunningham, V. L. Metrics to 'green' chemistry-which are the best? Green Chem. 2002, 4, 521527.

(3) Hernández, J. G.; Bolm, C. Altering Product Selectivity by Mechanochemistry. J. Org. Chem. 2017, 82, 4007-4019.

(4) James, S. L.; Adams, C. J.; Bolm, C.; Braga, D.; Collier, P.; Friščić, T.; Grepioni, F.; Harris, K. D. M.; Hyett, G.; Jones, W.; Krebs, A.; Mack, J.; Maini, L.; Orpen, A. G.; Parkin, I. P.; Shearouse, W. C.; Steed, J. W.; Waddell, D. C. Mechanochemistry: opportunities for new and cleaner synthesis. Chem. Soc. Rev. 2012, 41, 413-447.

(5) Staab, H. A. Reaktionsfähige Heterocyclische Diamide Der Kohlensäure. Justus Liebigs Ann. Chem. 1957, 609, 75-83.

(6) Paul, R.; Anderson, G. W. N,N'-Carbonyldiimidazole, a New Peptide Forming Reagent. J. Am. Chem. Soc. 1960, 82, 4596-4600.

(7) Armstrong, A.; Li, W. In e-EROS Encyclopedia of Reagents for Organic Synthesis, 2007.

(8) Verma, S. K.; Ghorpade, R.; Pratap, A.; Kaushik, M. P. Solvent free, $\mathrm{N}, \mathrm{N}^{\prime}$-carbonyldiimidazole $(\mathrm{CDI})$ mediated amidation. Tetrahedron Lett. 2012, 53, 2373-2376.

(9) For an example of reaction of CDI in EtOAc, see Dunn, P. J.; Hoffmann, W.; Kang, Y.; Mitchell, J. C.; Snowden, M. J. A Comparison of Catalysts to Promote Imidazolide Couplings Including the Identification of 2-Hydroxy-5-nitropyridine as a New, Safe, and Effective Catalyst. Org. Process Res. Dev. 2005, 9, 956-961.

(10) Métro, T.-X.; Bonnamour, J.; Reidon, T.; Sarpoulet, J.; Martinez, J.; Lamaty, F. Mechanosynthesis of amides in the total absence of organic solvent from reaction to product recovery. Chem. Commun. 2012, 48, 11781-11783.

(11) For an example of scaled-up organic reactions in a ball-mill, see Kaupp, G.; Schmeyers, J.; Naimi-Jamal, M. R.; Zoz, H.; Ren, H. Reactive milling with the Simoloyer ${ }^{\circledR}$ : environmentally benign quantitative reactions without solvents and wastes. Chem. Eng. Sci. 2002, 57, 763-765.

(12) Métro, T.-X.; Bonnamour, J.; Reidon, T.; Duprez, A.; Sarpoulet, J.; Martinez, J.; Lamaty, F. Comprehensive Study of the OrganicSolvent-Free CDI-Mediated Acylation of Various Nucleophiles by Mechanochemistry. Chem. - Eur. J. 2015, 21, 12787-12796.

(13) O'Connor, P.; Wolinsky, J. S.; Confavreux, C.; Comi, G.; Kappos, L.; Olsson, T. P.; Benzerdjeb, H.; Truffinet, P.; Wang, L.;
Miller, A.; Freedman, M. S. Randomized Trial of Oral Teriflunomide for Relapsing Multiple Sclerosis. N. Engl. J. Med. 2011, 365, 12931303.

(14) Tirapegui, C.; Acevedo-Fuentes, W.; Dahech, P.; Torrent, C.; Barrias, P.; Rojas-Poblete, M.; Mascayano, C. Easy and rapid preparation of benzoylhydrazides and their diazene derivatives as inhibitors of 15-lipoxygenase. Bioorg. Med. Chem. Lett. 2017, 27, 1649-1653.

(15) Mocci, R.; Luca, L. D.; Delogu, F.; Porcheddu, A. An Environmentally Sustainable Mechanochemical Route to Hydroxamic Acid Derivatives. Adv. Synth. Catal. 2016, 358, 3135-3144.

(16) Kumar, V.; Giri, S. K.; Venugopalan, P.; Kartha, K. P. R Synthesis of Cross-Linked Glycopeptides and Ureas by a Mechanochemical, Solvent-Free Reaction and Determination of Their Structural Properties by TEM and X-ray Crystallography. ChemPlusChem 2014, 79, 1605-1613.

(17) Métro, T.-X.; Gervais, C.; Martinez, A.; Bonhomme, C.; Laurencin, D. Unleashing the Potential of $17 \mathrm{O}$ NMR Spectroscopy Using Mechanochemistry. Angew. Chem., Int. Ed. 2017, 56, 68036807.

(18) Lanzillotto, M.; Konnert, L.; Lamaty, F.; Martinez, J.; Colacino, E. Mechanochemical 1,1'-Carbonyldiimidazole-Mediated Synthesis of Carbamates. ACS Sustainable Chem. Eng. 2015, 3, 2882-2889.

(19) Konnert, L.; Dimassi, M.; Gonnet, L.; Lamaty, F.; Martinez, J.; Colacino, E. Poly(ethylene) glycols and mechanochemistry for the preparation of bioactive 3,5-disubstituted hydantoins. RSC Adv. 2016, 6, 36978-36986.

(20) Konnert, L.; Gonnet, L.; Halasz, I.; Suppo, J.-S.; de Figueiredo, R. M.; Campagne, J.-M.; Lamaty, F.; Martinez, J.; Colacino, E. Mechanochemical Preparation of 3,5-Disubstituted Hydantoins from Dipeptides and Unsymmetrical Ureas of Amino Acid Derivatives. J. Org. Chem. 2016, 81, 9802-9809.

(21) Mascitti, A.; Lupacchini, M.; Guerra, R.; Taydakov, I.; Tonucci, L.; d'Alessandro, N.; Lamaty, F.; Martinez, J.; Colacino, E. Poly(ethylene glycol)s as grinding additives in the mechanochemical preparation of highly functionalized 3,5-disubstituted hydantoins. Beilstein J. Org. Chem. 2017, 13, 19-25.

(22) Engstrom, K. M.; Sheikh, A.; Ho, R.; Miller, R. W. The Stability of N,N-Carbonyldiimidazole Toward Atmospheric Moisture. Org. Process Res. Dev. 2014, 18, 488-494.

(23) Bonnamour, J.; Métro, T.-X.; Martinez, J.; Lamaty, F. Environmentally benign peptide synthesis using liquid-assisted ballmilling: application to the synthesis of Leu-enkephalin. Green Chem. 2013, 15, 1116-1120.

(24) Vaidyanathan, R.; Kalthod, V. G.; Ngo, D. P.; Manley, J. M.; Lapekas, S. P. Amidations Using N,N'-Carbonyldiimidazole: Remarkable Rate Enhancement by Carbon Dioxide. J. Org. Chem. 2004, 69, 2565-2568.

(25) Dunetz, J. R.; Magano, J.; Weisenburger, G. A. Large-Scale Applications of Amide Coupling Reagents for the Synthesis of Pharmaceuticals. Org. Process Res. Dev. 2016, 20, 140-177. 